\title{
An Unusual Presentation of Endocarditis Caused by Staphylococcus warneri
}

\author{
Ganesh D. Kini ${ }^{1, *}$, Ketan Patel ${ }^{1}$, Addison R. Parris ${ }^{2}$ and Jane S. Tang ${ }^{3}$. \\ ${ }^{I}$ Department of Medicine and ${ }^{2}$ Department of Infectious Diseases, Rockingham Memorial Hospital, Harrisonburg, \\ Virginia; ${ }^{3}$ Noblis Falls Church, Virginia
}

\begin{abstract}
Staphylococcus warneri does not generally cause serious infections in humans. We report a case of endocarditis in a healthy individual with no known past medical history. S. warneri was identified in her blood cultures and echocardiographic evidence confirmed the diagnosis of bacterial endocarditis. There was no apparent cause for her infection, and risk factors such as invasive treatment or medical implant were not present. This rare clinical presentation illustrates the importance of not overlooking low virulence species of Staphylococcus, as they can potentially serve as opportunistic etiological agents for endocarditis, especially among the elderly population.
\end{abstract}

Keywords: Staphylococcus warneri, coagulase negative staphylococci, endocarditis, immunocompetent.

\section{INTRODUCTION}

Staphylococcus warneri is part of the normal microbial flora on the skin [1-3] and generally is not considered pathogenic [4]. As a member of the coagulase negative Staphylococcus (CoNS) group, this organism does not raise as much concern as $S$. epidermidis, which has been associated with an increasing number of nosocomial infections [2-4]. S. warneri occasionally caused septicemia, osteomyelitis and other types of infections [2-7]. Most of the clinical cases due to $S$. warneri infection have occurred in immunocompromised patients with invasive treatments or medical device implants. $S$. warneri has rarely been reported to cause disease in healthy people $[4,6]$.

In the past few years, several endocarditis cases were traced to $S$. warneri as the etiological agent. These incidences often involved certain medical devices, such as the insertion of prosthetic heart valves or shunts in immunosuppressed patients $[1,6]$. One clinical case described an unusual endocarditis from an immunocompetent individual who had a native aortic valve that was contaminated with $S$. warneri, and a prosthetic lumbar disc implanted 26 months prior was suggested as the probable source of infection [5]. Apparently there was precedence of another endocarditis case which developed one year after the implantation of a hip prosthesis [6]. These observations led the authors to caution that $S$. warneri could potentially cause endocarditis in patients with foreign body implants. In addition, endocarditis cases involving community-acquired native valve or prosthetic valve replacements have been reported $[8,9]$.

\section{CASE PRESENTATION}

A 78-year-old Caucasian woman with no known past medical history presented to the emergency department with complaints of bilateral lower extremity edema and erythema

*Address correspondence to this author at the Department of Medicine, Rockingham Memorial Hospital, 2010 Health Campus Drive, Harrisonburg, VA 22801, USA; Tel: 540-689-1100; Fax: 540-689-1119;

E-mail: gkini@ rhcc.com with a clear serous discharge. The patient first noticed some small blisters on her left lower extremity which subsequently opened with some clear serous drainage. She also experienced progressively worsening swelling first of the left lower extremity and then her right lower extremity which prompted her to come into the hospital. Except for a cough, the patient had no other symptoms.

Preliminary evaluation at the emergency department indicated the patient had cardiomegaly and bilateral pleural effusions with a BNP of 1260 . She also had atrial fibrillation with rapid ventricular response. The patient was not complaining of any chest pain, fevers, chills, nausea, vomiting or diarrhea. Her chest x-ray showed evidence of cardiac enlargement with interstitial densities and bilateral air-space processes possibly associated with asymmetric pulmonary edema. Large bilateral pleural effusions were noted, with the left greater than the right side. Incidentally, some evidence indicating a compression fracture of indeterminate age involving the upper lumbar/lower dorsal spine was observed.

The patient had no known medical history. In fact, she had not been seen by a physician for the last 15 years. Besides taking aspirin occasionally, she was not on any medications. She did not use alcohol, tobacco or illicit drugs. Physical examination did not reveal any specific concerns other than bilateral lower extremity edema.

CT angiogram of the chest showed large bilateral pleural effusions with bilateral lower lobe enhancing volume loss and worse on the left. Cardiomegaly was noted and there was no evidence for a pulmonary embolus. In addition, there was no evidence of deep venous thrombosis in the venous Doppler studies of her lower extremities bilaterally.

Echocardiogram indicated the left ventricle with some mild hypertrophy. Systolic function was normal with an ejection fraction estimated at about $60 \%$. The right ventricle was mildly enlarged and mildly hypokinetic. The left atrium showed marked enlargement and the right atrium was moderately enlarged as well. The aortic valve was mildly sclerotic and trileaflet. The pulmonic valve was unremarkable. 
The mitral valve showed flail motion of the posterior leaflet and there was a small mobile echo seen in the left atrium which strongly indicated endocarditis as the cause for pulmonary edema. Doppler examination showed severe mitral regurgitation that tracked underneath the anterior leaflet along the interatrial septum. There was mild aortic insufficiency. The inferior vena cava was dilated indicating elevated right atrial pressure and the pulmonary systolic pressure was estimated at about $50 \mathrm{mmHg}$.

Gram positive cocci in diploids, tetrads, and clusters were isolated from three sets of blood cultures. The organism was catalase positive and produced a negative coagulase reaction by Slidex $^{\mathrm{TM}}$ (BioMérieux, NC). The Vitek 2 GP card (BioMérieux, MO) identified the bacterium as Staphylococcus warneri. It produced acid from trehalose and lacked phosphatase enzyme, two key characteristics that differentiate this species from S. epidermidis - one of the most recognized CoNS [1].

The patient was initially treated with Unasyn, 3 grams IV every 6 hours for presumed bilateral lower extremity cellulitis. After the diagnosis of bacterial endocarditis was made and supported by the echo-cardiographic results, the patient was started on nafcillin with gentamicin following consultation with the infectious diseases specialist. In addition, congestive heart failure was treated initially with lasix IV and subsequently with lasix orally. Atrial fibrillation was rate controlled with diltiazem. Gentamicin was later discontinued due to acute renal failure, and the patient was discharged with a PICC line for treatment with 2 grams Nafcillin intravenously every 4 hours for a total of 6 weeks. Repeat blood cultures were negative for 48 hours prior to discharge.

\section{COMMENTS}

The most common causative bacterial agents for infective endocarditis (IE) are viridans streptococci and they have been recovered from a significant portion of IE patients (up to 50\%) [10-12]. Aside from viridans streptococci, different species of Staphylococcus have been reported to cause IE. Among them, S. aureus was the most frequently isolated species [10]. Other bacteria associated with endocarditis are presented in the Table 1 below [13].

Table 1. Bacteria Associated with Infective Endocarditis

\begin{tabular}{|l|l|}
\hline Common bacterial agents & Less common bacterial agents \\
\hline Gram positive organisms & Gram positive organisms \\
\hline Viridan streptococci & Abiotrophia \\
\hline Staphylococcus aureus & Gemella \\
\hline Coagulase negative staphylococci & Nocardia \\
\hline Enterococci & Clostridia \\
\hline Gram negative organisms & Gram negative organisms \\
\hline Haemophilus & Bartonella \\
\hline Actinobacillus & Legionella \\
\hline Cardiobacterium & Coxiella burnetii \\
\hline Eikenella & Pasteurella \\
\hline Kingella & Brucella \\
\hline
\end{tabular}

Several endocarditis cases were traced to $S$. warneri as the etiological agent and they generally involved foreign body implants or medical device insertions in patients. These observations indicate $S$. warneri has the potential to cause serious invasive infections [8]. The case reported here is a rare presentation of endocarditis which occurred in a fairly healthy individual without an apparent source of infection. The patient has not sought medical treatment for at least 15 years. High-risk conditions for endocarditis, such as congenital heart disease, IV drug use, implants, central venous catheters, as well as various invasive procedures were not found in the patient's medical history. The only possible predisposition to illness is her age, and one plausible mode of etiological agent introduction is via the oral cavity. A recent study reported $S$. warneri as one of the staphylococci identified in saliva of health adults. Although its occurrence rate was relatively low when compared to other Staphylococcus species, results of the study suggested oral cavity as a route of entry for infective endocarditis [10]. Furthermore, the frequency of staphylococci recovered from saliva increases with age [14], and most patients suffering from endocarditis are elderly [15]. Thus, $S$. warneri can potentially infect older population who are in relatively good health.

As medical practices continue to involve more invasive procedures, the importance of detecting CoNS members has been communicated in the clinical community $[4,16$, 17]. Among the concerns is the ability of these bacteria to resist antibiotics and interfere with host defense mechanism $[2,16,17]$. Although $S$. warneri is often innocuous and susceptible to antimicrobial treatments, it should not be dismissed as a skin flora contaminant when isolated from blood cultures.

In conclusion, clinicians should be cognizant of $S$. warneri as a possible low virulence but invasive pathogen. Its ability to cause infection is not limited to patients with implants or catheters. As with many other organisms with low virulence potential, it may pose as a risk factor for elderly patients susceptible to different types of infections.

\section{ACKNOWLEDGEMENT}

The author would like to acknowledge Jennifer Clevinger, Clinical Microbiology Senior Technologist at Rockingham Memorial Hospital, for microbiological testing and data.

\section{REFERENCES}

[1] Dan M, Marien GJ, Goldsand G. Endocarditis caused by Staphylococcus warneri on a normal aortic valve following vasectomy. Can Med Assoc J 1984; 131: 211-3.

[2] Bannerman TL. Staphylococcus, Micrococcus, and Other Catalasepositive Cocci that Grow Aerobically. In: Murray PR, Ed. Manual of Clinical Microbiology, $8^{\text {th }}$ ed. Washington DC, ASM Press 2003; pp. 384-404.

[3] Leighton PM, Little JA. Identification of coagulase-negative staphylococci isolated from urinary tract infection. Am J Clin Pathol 1986; 85: 92-5.

[4] Tufariello AM, Lowy FD. Microbiology, pathogenesis, and epidemiology of coagulase-negative staphylococci. UpToDate 2008. Available from: http: //www.uptodate.com/patients/content/topic.do? topicKey=gram_pos/3019

[5] Stollberger C, Wechsler-Fordos A, Geppert F, et al. Staphylococcus warneri endocarditis after implantation of a lumbar disc prosthesis in an immunocompetent patient. J Infect 2006; 52: e15-8. 
[6] Wood CA, Sewell, DL, Strausbaugh, LJ. Vertebral osteomyelitis and native valve endocarditis caused by Staphylococcus warneri. Diagn Microbiol Infect Dis 1989; 12: 251-3.

[7] Kamath U, Singer C, Isenberg HD. Clinical significance of Staphylococcus warneri bacteremia. J Clin Microbiol 1992; 30: 261-4.

[8] Wood CA. Significant infection caused by Staphylococcus warneri. J Clin Microbiol 1992; 30: 2216-7.

[9] Abgrall S, Meimoun P, Buu-Hoi A, Couetil JP, Gutmann L, Mainardi JL. Early prosthetic valve endocarditis due to Staphylococcus warneri with negative blood culture. J Infect 2001; 42: 1669.

[10] Ohara-Nemoto Y, Haraga H, Kimura S, Nemoto, TK. Occurrence of staphylococci in the oral cavities of healthy adults and nasal-oral trafficking of the bacteria. J Med Microbiol 2008; 57: 95-9.

[11] Strom BL, Abrutyn E, Berlin JA, et al. Dental and cardiac risk factors for infective endocarditis. A population-based, case-control study. Ann Intern Med 1998; 129: 761-9.
[12] Di Filippo S, Delahaye F, Semiond B, et al. Current patterns of infective endocarditis in congenital heart disease. Heart 2006; 92: 1490-5.

[13] Brouqui P, Raoult D. Endocarditis due to rare and fastidious bacteria. Clin Microbiol Rev 2001; 14: 177-207.

[14] Hoen B, Alla F, Selton-Suty C, et al. Changing profile of infective endocarditis. Results of a 1 year survey in France. JAMA 2002; 288: 75-81

[15] Percival RS, Challacombe SJ, Marsh, PD. Age-related microbiological changes in the salivary and plaque microflora of healthy adults. J Med Microbiol 1991; 35: 5-11.

[16] Centers for Disease Control and Prevention. Laboratory detection of coagulase negative Staphylococcus species with decreased susceptibility to the glycopeptides vancomycin and teicoplanin. 1999. Available from: http: //www.cdc.gov/ncidod/dhqp/ar_lab_IntStaph. html.

[17] Kloos WE, Bannerman TL. Update on clinical significance of coagulase-negative staphylococci. Clin Microbial Rev 1994; 7: 117-40.

(c) Kini et al.; Licensee Bentham Open.

This is an open access article licensed under the terms of the Creative Commons Attribution Non-Commercial License (http://creativecommons.org/licenses/ by-nc/3.0/) which permits unrestricted, non-commercial use, distribution and reproduction in any medium, provided the work is properly cited. 